\title{
ESTERCO DE GADO LEITEIRO ASSOCIADO À ADUBAÇÃO MINERAL E SUA INFLUÊNCIA NA FERTILIDADE DE UM LATOSSOLO SOB PLANTIO DIRETO ${ }^{(1)}$
}

\author{
José Carlos Peixoto Modesto da Silva ${ }^{(2)}$, Antônio Carlos Vargas Motta ${ }^{(3)}$, \\ Volnei Pauletti $^{(3)}$, Cristina Mattos Veloso ${ }^{(4)}$, Nerilde Favaretto ${ }^{(3)}$, Milena \\ Barcellos $^{(5)}$, André Soares de Oliveira ${ }^{(6)} \&$ Luiz Fernando Costa e Silva ${ }^{(7)}$
}

\begin{abstract}
RESUMO
A produção de gado de leiteiro no sul do Brasil ocorre em grande parte em sistema de semi ou completo confinamento dos animais, o que gera uma produção significativa de resíduos. Os resíduos gerados vêm sendo utilizados como única fonte de nutrientes ou associados a fontes minerais na produção de grãos e silagem, mas sua influência nas características de solo não tem sido bem caracterizada. Este trabalho foi realizado com o objetivo de avaliar a influência de doses de adubação com esterco líquido de gado leiteiro, associadas com adubação mineral, sobre os níveis de $\mathrm{P}, \mathrm{K}$, C e condutividade elétrica (CE) de um Latossolo Bruno, em sistema plantio direto e rotação de culturas de inverno e verão (sorgo/aveia-preta/ milho/azevém/milho/azevém), para produção de silagem, nas camadas de $\mathbf{0}-\mathbf{5}, \mathbf{5}-\mathbf{1 0}$, 10-30, 30-50 e 50-80 cm de profundidade. Os tratamentos foram distribuídos em três blocos casualizados, divididos em 12 parcelas cada bloco, em arranjo fatorial $3 \times$ 4, sendo três níveis de adubação mineral (0, 50 e $100 \%$ da dose recomendada para as culturas) e quatro níveis de adubação orgânica $\left(0,30,60\right.$ e $90 \mathrm{~m}^{3} \mathrm{ha}^{-1}$ ano $\left.^{-1}\right)$. A adubação mineral proporcionou maiores valores de $\mathbf{P}$ disponível (Mehlich-1 e resina) até a profundidade de $10 \mathrm{~cm}$, dada a adição em profundidade pela plantadeira, enquanto, com esterco aplicado em superfície, os maiores valores
\end{abstract}

\footnotetext{
(1) Recebido para publicação em março de 2008 e aprovado em janeiro de 2010.

${ }^{(2)}$ Doutor em Zootecnia pela Universidade Federal de Viçosa - UFV. CEP 36570-000 Viçosa (MG). E-mail: jcpmodesto@yahoo.com.br

(3) Professores Adjuntos do Departamento de Solos e Engenharia Agrícola, Universidade Federal do Paraná - UFPR. Rua dos Funcionários 1540, CEP 80035-050 Curitiba (PR). E-mails: mottaacv@ufpr.br; vpauletti@ufpr.br; nfavaretto@ufpr.br

(4) Professora Adjunta do Departamento de Zootecnia, Universidade Federal de Viçosa - UFV. CEP 36570-000 Viçosa (MG). E-mail: cristina.veloso@ufv.br

(5) Mestre em Ciência do Solo pela Universidade Federal do Paraná - UFPR. E-mail: milena_barcellos@yahoo.com.br

(6) Professor Adjunto do Departamento de Zootecnia, Universidade Federal de Mato Grosso - UFMT. Av. Fernando Corrêa da Costa 2367, Bairro Boa Esperança, CEP 78060-900 Cuiabá (MT). E-mail: andresoli@uol.com.br

(7) Mestrando em Zootecnia, UFV. E-mail: lfcostasilva@yahoo.com.br
} 
ficaram restritos à profundidade de $0-5 \mathrm{~cm}$. Constatou-se esgotamento de $\mathrm{K}$ ao longo do perfil devido à elevada extração via silagem, tendo o esterco líquido aplicado uma relação linear com teor de $\mathrm{K}$ disponível, em todas as profundidades analisadas. A ausência de efeito da adubação mineral sobre o $\mathrm{K}$ deve-se, provavelmente, à baixa dose de $\mathrm{K}$ adicionada. Foi observada relação direta entre doses de esterco e teores de C orgânico na camada de 0-5 cm, o mesmo não sendo observado quando do uso de adubo mineral. Baixos valores de CE sugerem grande exportação de nutrientes pela produção de silagem. Os resultados obtidos indicam que não houve movimentação significativa de $\mathbf{P}$ no perfil do Latossolo, mesmo após seis anos sob altas doses de esterco de gado leiteiro, e que o $\mathrm{K}$ aplicado via esterco e adubação mineral não é suficiente para manter os níveis deste nutriente em sistemas em que a extração de K pela silagem é alta. Nessas situações, o aumento dos teores de C orgânico é restrito à camada superficial do solo em plantio direto e diretamente relacionado com a dose de esterco aplicada.

Termos de indexação: adubação orgânica, fertilizante, nutriente do solo.

\title{
SUMMARY: INFLUENCE OF DAIRY CATTLE MANURE ASSOCIATED WITH MINERAL FERTILIZER ON SOIL FERTILITY OF AN OXISOL UNDER NO-TILLAGE
}

\begin{abstract}
Dairy cattle in South Brazil is raised mainly in a system based on partial or total confinement of the animals, generating a significant amount of residues. The residues have been used as the only nutrient source or associated to mineral sources in grain or silage production. Their effect on soil characteristics has however been little explored. This study was conducted to evaluate the influence of fertilization rates with liquid dairy cattle manure associated with mineral fertilizer on $P, K, C$, and electrical conductivity (EC) levels of a Brown Oxisol, under no-tillage and winter and summer crop rotation (sorghum/black-oat/corn/ryegrass /corn / ryegrass) for silage production, in the layers $0-5,5-10,10-30,30-50$ and 50-80 cm. The treatments were distributed in three randomized blocks, each divided in 12 plots, in a $3 \times 4$ factorial arrangement, with three mineral fertilizer levels (0, 50 and $100 \%$ of the dose recommended for the crops) and four organic fertilizer levels (0, 30, 60, and $90 \mathrm{~m}^{3} \mathrm{ha}^{-1}$ year-1). Mineral fertilizer resulted in higher values of available $P$ (Mehlich-1 and resin) to a depth of $10 \mathrm{~cm}$, due to the fertilizer application in depth by the planter, whereas the highest values under manure surface application were restricted to the $0-5 \mathrm{~cm}$ layer. Potassium depletion was observed along the soil profile due to high extraction by silage, and a linear ratio with available K level could be applied in the case of liquid manure at all depths. The lack of effect by the mineral fertilizer on $K$ is probably due to the low rate of $K$ application. A direct relationship was observed between manure rates and organic $C$ concentration in the $0-5 \mathrm{~cm}$ layer, unlike in the case of mineral fertilizer. Low EC values were obtained suggesting high nutrient exportation by silage production. Results indicate that there was no significant $P$ migration in the Oxisol profile, even after six years of dairy cattle manure application at high rates, and that $K$ applied via manure and mineral fertilizer is insufficient to maintain the levels of this nutrient in systems where K extraction by silage is high. In these situations, increased levels of organic $C$ are restricted to the soil surface layer in no-tillage and directly related to the manure rate.
\end{abstract}

Index terms: organic fertilization, fertilizer, soil nutrient.

\section{INTRODUÇÃO}

O uso de esterco, utilizado como adubo na produção de alimentos e forragem, vem aumentando com a intensificação da produção de animais em sistemas confinados, constituindo-se numa fonte de nutrientes para as plantas, o que pode diminuir os custos de produção (Assmann et al., 2007).

O conhecimento da dinâmica dos nutrientes no solo a partir da superfície, onde os fertilizantes são depositados no sistema plantio direto, é fundamental para estabelecer ajustes na recomendação de adubos 
e corretivos. As maiores dúvidas, que geram insegurança quando da utilização de esterco na adubação, estão ligadas aos efeitos de longo prazo que venham a ocorrer na condição de não revolvimento do solo após o uso intensivo por vários anos de esterco. $\mathrm{O}$ melhor entendimento das modificações nos atributos químicos do solo, decorrentes do uso de esterco na adubação, pode fornecer subsídios para produção em bases sustentáveis, sem comprometer o ambiente (Scherer et al., 2007).

Os nutrientes adicionados por adubação em sistema plantio direto, principalmente aqueles com menor mobilidade, como é o caso do $\mathrm{P}$, permanecem nas camadas mais superficiais do solo, criando gradientes de concentração em relação às camadas inferiores (Falleiro et al., 2003; Scherer et al., 2007). Contudo, o transporte do $\mathrm{P}$, da camada superficial para horizontes inferiores do perfil do solo, tem sido desprezado devido à facilidade com que ele se precipita com outros nutrientes na solução do solo ou é adsorvido (Ryden et al., 1973; Sims et al., 1998).

A adubação com esterco ou fertilizantes químicos pode levar a acréscimos de K no solo (Elrashidi, 1999). Todavia, quando da produção de silagem, especialmente de gramíneas, a exportação de K, do solo para a planta, pode ser superior ao adicionado pelo esterco ou fertilizante mineral, propiciando, quando não manejado adequadamente, em vez de acúmulo de K, esgotamento do elemento no solo (Vitosh et al., 1973; Kaminski et al., 2007), fazendo com que seja muito importante definir qual o melhor nível de adubação orgânica e mineral para obtenção de melhores rendimentos das culturas (Kaminski et al., 2007).

O uso de esterco combinado com adubação mineral tem sido uma estratégia de manejo importante para melhoria da qualidade do solo (Leite et al., 2003), promovendo elevação do teor de $\mathrm{C}$ no solo, quando se adiciona esterco em doses relativamente elevadas ou por tempo prolongado (Tiarks et al., 1974; Meek et al., 1982; Schjonning et al., 1994). Modificaçôes dos teores de cátions e ânions em solução levam à mudança na condutividade elétrica, sendo esta muito utilizada para verificar alterações e mobilidade de elementos em profundidade (Culley et al., 1981; Josan et al., 2005).
Desse modo, o objetivo deste trabalho foi analisar a dinâmica do $\mathrm{P}, \mathrm{K}, \mathrm{C}$ e condutividade elétrica do solo, após seis anos de aplicação periódica de diferentes doses de esterco líquido de gado leiteiro combinadas com fertilizantes minerais.

\section{MATERIAL E MÉTODOS}

O experimento foi conduzido na Fazenda CapãoAlto, no município de Castro-PR, no período de março de 1997 a julho de 2003, em área experimental da Fundação ABC (Arapoti, Batavo e Castrolanda), na região fisiográfica denominada Terceiro Planalto Paranaense, entre as coordenadas de $24^{\circ} 50$ ' de latitude sul e $49^{\circ} 50$ ' de longitude oeste, com altitude média de $900 \mathrm{~m}$. O clima da região, segundo a classificação climática de Köppen, é do tipo Cfb, clima temperado propriamente dito, registrando temperaturas médias nos meses mais frios entre $13 \mathrm{e}$ $14^{\circ} \mathrm{C}$ e nos meses mais quentes entre 23 e $24^{\circ} \mathrm{C}$, sem estação seca definida, com pluviosidade anual entre 1.400 e $1.600 \mathrm{~mm}$, sendo a média no trimestre mais seco entre 250 e $350 \mathrm{~mm}$ e, no trimestre mais chuvoso, entre 400 e $500 \mathrm{~mm}$. A umidade relativa média do ar apresenta-se entre 70 e $75 \%$, e a evapotranspiração média anual, entre 900 e $1.000 \mathrm{~mm}$. A vegetação original é caracterizada por campo subtropical dos Campos Gerais do Paraná (IAPAR, 2000).

O solo, de acordo com a classificação da Embrapa (1999), é caracterizado como Latossolo Bruno distroférrico textura argilosa fase campo subtropical, com relevo suavemente ondulado. As características químicas médias do solo, por ocasião da instalação do experimento, podem ser observadas no quadro 1. Convém salientar que a área apresentava, anteriormente ao período de estudo, histórico de 16 anos de adubação orgânica com esterco, em sistema plantio direto, e intensa rotação de culturas típicas da região, tendo sido cultivados, no verão, principalmente milho e soja e, no inverno, cereais e forrageiras. Esses fatores são determinantes da elevada fertilidade apresentada pelo solo quando da instalação do experimento.

Quadro 1. Parâmetros químicos do solo da área de estudo quando da instalação do experimento

\begin{tabular}{|c|c|c|c|c|c|c|c|c|c|}
\hline Profundidade & $\mathrm{pH}\left(\mathrm{CaCl}_{2}\right)$ & $\mathbf{H}+\mathbf{A l}$ & $\mathrm{Al}^{3+}$ & $\mathbf{K}^{+}$ & $\mathrm{Ca}^{2+}$ & $\mathrm{Mg}^{2+}$ & $\mathbf{P}$ & $\mathbf{C}$ & $\mathbf{V}$ \\
\hline $\mathrm{cm}$ & & & 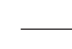 & $-\mathrm{c}$ & ${ }_{c} \mathrm{dm}^{-3}$ & 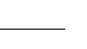 & $\mathrm{mg} \mathrm{dm} \mathrm{m}^{-3}$ & $\mathrm{~g} \mathrm{~kg}^{-1}$ & $\%$ \\
\hline $0-5$ & 5,3 & 4,54 & 0,0 & 0,7 & 2,8 & 1,9 & 40,3 & 31,3 & 53,9 \\
\hline $5-10$ & 5,1 & 5,41 & 0,0 & 0,5 & 2,1 & 1,3 & 19,1 & 25,0 & 42,2 \\
\hline $10-30$ & 5,2 & 4,12 & 0,0 & 0,4 & 2,4 & 1,3 & 8,8 & 21,1 & 49,5 \\
\hline $30-50$ & 5,2 & 5,11 & 0,0 & 0,3 & 1,9 & 1,1 & 3,7 & 19,4 & 39,1 \\
\hline $50-80$ & 4,8 & 5,57 & 0,1 & 0,2 & 1,0 & 0,8 & 2,0 & 16,4 & 25,3 \\
\hline
\end{tabular}


Os tratamentos foram distribuídos em três blocos casualizados com três repetições, divididos em 12 parcelas cada bloco, em arranjo fatorial $3 \times 4$, contendo três níveis de adubação mineral (0, 50 e $100 \%$ da dose recomendada para as culturas) e quatro níveis de adubação orgânica (0, 30, 60 e $90 \mathrm{~m}^{3} \mathrm{ha}^{-1}$ ano $\left.^{-1}\right)$. Quando da implantação do experimento, foi feita correção do solo com calcário dolomítico aplicado a lanço na dose de $1 \mathrm{t} \mathrm{ha}^{-1}$ e, após 30 dias, realizada a semeadura da cultura do sorgo, a qual foi a primeira cultura do período experimental.

A adubação mineral foi baseada na análise inicial do solo, e a quantidade de adubo mineral correspondente a $100 \%$ do recomendado para as culturas, sem aplicação de esterco, encontra-se no quadro 2. O milho e o sorgo foram semeados com mecanismo sulcador do tipo haste da empresa Semeato, também conhecido como guilhotina ${ }^{\circledR}$ (Semeato, 2006), com medidas de 2,5 cm na ponteira, $4,0 \mathrm{~cm}$ na maior largura da haste e $11,0 \mathrm{~cm}$ de profundidade de trabalho. A aveia e o azevem foram plantadas com disco duplo defasado composto por dois discos, sendo um de $339 \mathrm{~mm}$ e outro de $363 \mathrm{~mm}$, com aproximadamente $8,0 \mathrm{~cm}$ de profundidade de trabalho. Os dois mecanismos sulcadores trabalharam alinhados ao sulco de deposição das sementes; no primeiro o adubo foi colocado a aproximadamente 5 a $6 \mathrm{~cm}$ abaixo das sementes, enquanto no segundo (disco duplo) ele foi depositado a aproximadamente 2 a $3 \mathrm{~cm}$ abaixo delas.

O esterco utilizado foi proveniente da criação de gado leiteiro, em sistema intensivo de manejo em galpões de confinamento (Free stall), sendo composto pela mistura de fezes e urina dos animais, além de outros materiais provenientes do processo criatório, como água desperdiçada nos bebedouros, água de higienização e restos de alimentos. Os dejetos foram armazenados em esterqueiras, próximas ao galpão de confinamento, e homogeneizados diariamente. $\mathrm{O}$ transporte do esterco foi feito por meio de trator, com vagão contendo rosca sem fim, que permite a homogeneização do esterco quando este está sendo transportado para o campo. Foram utilizadas doses anuais de 0, 30, 60 e $90 \mathrm{~m}^{3} \mathrm{ha}^{-1}$, aplicadas metade nas forrageiras de inverno e o restante nas de verão, manualmente em superfície e sem incorporação. As características do esterco, de três amostras coletadas no período do experimento, são apresentadas no quadro 3. A rotação de culturas e cultivares está apresentada no quadro 4, com os respectivos anos de plantio. As plantas foram cortadas e ensiladas, para posterior fornecimento no cocho aos animais.

A coleta de solo foi feita no inverno de 2003, em cinco profundidades (0-5, 5-10, 10-30, 30-50 e 50$80 \mathrm{~cm}$ ); nas profundidades de 0-5, 5-10 e 10-30 cm, foram retiradas 15 subamostras simples para compor uma amostra composta, utilizando trado calador. Nas profundidades de 30-50 e 50-80 cm foram retiradas cinco subamostras simples com trado holandês, as quais foram misturadas para compor uma amostra composta. A amostragem foi realizada aleatoriamente nas parcelas e com os cuidados recomendados para a não contaminação das amostras superiores, como a limpeza de profundidade de amostras no sentido das profundidades maiores para as menores.

As análises químicas para o $\mathrm{P}$ e $\mathrm{K}$, usando o extrator Mehlich-1, foram realizadas no laboratório de química e fertilidade do solo do Departamento de Solos e Engenharia Agrícola da Universidade Federal do Paraná, com base em método descrito em Marques \& Motta (2003). A análise do P extraído pelo método da resina trocadora de íons foi realizada pelo laboratório da Fundação ABC, em Castro-PR, segundo método descrito por (Raij et al., 1986). As análises do carbono foram feitas pelo método volumétrico descrito por Quaggio \& Raij (1979), e para a condutividade elétrica utilizou-se o método descrito por Sonneveld et al. (1990).

Os dados foram submetidos à análise de variância e regressão, adotando-se nível de $5 \%$ para o erro tipo I, utilizando-se o pacote estatístico SAS (SAS, 1996). Nos casos em que a interação adubação mineral $\times$ adubação orgânica não foi significativa, efetuou-se o estudo de regressão de primeiro e segundo graus, por meio do comando GLM.

Quadro 2. Quantidade de $\mathrm{N}, \mathrm{P}_{2} \mathrm{O}_{5}$ e $\mathrm{K}_{2} \mathrm{O}$ aplicada, correspondente a $100 \%$ da adubação mineral, no plantio e na cobertura nas culturas de verão e inverno

\begin{tabular}{|c|c|c|c|c|c|}
\hline \multirow{2}{*}{ Cultura } & \multicolumn{3}{|c|}{ Doses de plantio } & \multicolumn{2}{|c|}{ Doses de cobertura } \\
\hline & $\mathbf{N}$ & $\mathbf{P}_{2} \mathbf{O}_{5}$ & $\mathbf{K}_{2} \mathbf{O}$ & $\mathbf{N}$ & $\mathrm{K}_{2} \mathrm{O}$ \\
\hline & & & & & \\
\hline Sorgo & 30 & 60 & 60 & 90 & 0 \\
\hline Milho & 30 & 60 & 60 & 90 & 0 \\
\hline Aveia-Preta & 20 & 60 & 60 & 37,5 & 37,5 \\
\hline Azevém & 16 & 45 & 0 & 77,5 & 0 \\
\hline Total $(6 \text { anos })^{(1)}$ & 284 & 660 & 480 & 925 & 75 \\
\hline
\end{tabular}

(1) Calculado considerando as culturas de inverno e verão e os anos de cultivo. 
Quadro 3. Teores e quantidades totais médias de matéria seca (MS) e de nutrientes aplicados nos tratamentos com esterco de gado leiteiro

\begin{tabular}{|c|c|c|c|c|c|c|c|c|c|}
\hline Amostra & MS & $\mathbf{N}$ & $\mathbf{P}$ & $\mathbf{K}$ & $\mathbf{C a}$ & Mg & $\mathbf{F e}$ & $\mathrm{Cu}$ & $\mathrm{Zn}$ \\
\hline & \multicolumn{6}{|c|}{$-\mathrm{g} \mathrm{kg}-1^{-1}$} & \multicolumn{3}{|c|}{$-\mathrm{mg} \mathrm{kg}^{-1}$} \\
\hline 1 & $78^{(1)}$ & 20,0 & 9,1 & 40,0 & 21,0 & 13,0 & 2.600 & 105 & 510 \\
\hline 2 & 69 & 16,0 & 16,4 & 46,5 & nd & nd & nd & nd & nd \\
\hline 3 & 55 & 20,2 & 13,6 & 39,9 & nd & nd & nd & nd & nd \\
\hline Dose & MS & $\mathbf{N}$ & $\mathbf{P}_{2} \mathbf{O}_{5}$ & $\mathbf{K}_{2} \mathbf{O}$ & $\mathrm{CaO}$ & MgO & $\mathbf{F e}$ & $\mathrm{Cu}$ & $\mathrm{Zn}$ \\
\hline \multicolumn{10}{|l|}{$\mathrm{m}^{3} \mathrm{ha}^{-1}$} \\
\hline 30 & $2^{(2)}$ & 38 & 62 & 103 & 69 & 50,5 & 5,9 & 0,25 & 1,2 \\
\hline 60 & 4 & 76 & 124 & 206 & 138 & 101 & 11,7 & 0,50 & 2,4 \\
\hline 90 & 6 & 114 & 186 & 309 & 207 & 152 & 17,5 & 0,75 & 3,6 \\
\hline
\end{tabular}

$\overline{(1)}$ Teor de nutrientes em base seca. ${ }^{(2)}$ Quantidade média de nutrientes, calculada em função dos teores de três ou uma amostra do resíduo.

Quadro 4. Rotação de culturas de verão e inverno na área experimental, entre 1997 e 2003

\begin{tabular}{lll}
\hline \multirow{2}{*}{ Ano de plantio } & \multicolumn{1}{c}{ Forrageira } \\
\cline { 2 - 3 } & \multicolumn{1}{c}{ Verão } \\
\hline $1997 / 1998$ & Sorgo AG 2005 (Sorghum vulgare Pers.) & \multicolumn{1}{c}{ Inverno } \\
$1998 / 1999$ & Milho AG 9012 (Zea mays L.) & Azevém comum (Lolium multiflorum Lam.) \\
$1999 / 2000$ & Milho AG 9012 (Zea mays L.) & Azevém comum (Lolium multiflorum Lam.) \\
$2000 / 2001$ & Sorgo AG 2005 (Sorghum vulgare Pers.) & AveiaPreta comum (Avena strigosa Sereb) \\
$2001 / 2002$ & Milho AG 9012 (Zea mays L.) & Azevém comum (Lolium multiflorum Lam.) \\
$2002 / 2003$ & Milho AG 9012 (Zea mays L.) & Azevém comum (Lolium multiflorum Lam.) \\
\hline
\end{tabular}

\section{RESULTADOS E DISCUSSÃO}

No quadro 5 são mostrados os efeitos dos tratamentos sobre a significância da interação e do ajuste à regressão linear e quadrática dos parâmetros do solo avaliados, enquanto no quadro 6 são apresentadas as equações de regressão para $\mathrm{P}$ no extrator resina (P-resina), P no extrator Mehlich-1, $\mathrm{K}^{+}$trocável, carbono orgânico e condutividade elétrica, nas diversas profundidades do solo. Constatou-se que não houve interação entre a aplicação de esterco e a adubação mineral.

Os teores de $\mathrm{P}$ no solo apresentaram relação linear com as doses aplicadas de adubação mineral até a profundidade de $10 \mathrm{~cm}$ (Figura 1a,b), enquanto a relação linear proporcionada pela adubação orgânica ficou restrita à profundidade de $0-5 \mathrm{~cm}$, para ambos os extratores: P-resina e Mehlich-1 (Figura 1c,d). O aumento até a camada de $10 \mathrm{~cm}$ com adubação mineral era esperado, visto que as plantadeiras utilizadas adicionavam adubo abaixo de $5 \mathrm{~cm}$ de profundidade. Observa-se que, mesmo com teores muito altos de $\mathrm{P}$ nas camadas superficiais, pois os valores ficaram acima de $18 \mathrm{mg} \mathrm{dm}^{-3}$ de P Mehlich-1 (CQFSRS/SC,
2004), não houve significativa migração do nutriente para camadas inferiores do perfil. No entanto, esperava-se que a presença de grande número de bioporos, observados na área experimental, quando da amostragem do solo, pudesse auxiliar na movimentação de $\mathrm{P}$ em profundidade, visto que bioporos representam o principal mecanismo de movimentação de P no solo (Geohring et al., 2001; Motta, 2002).

Justifica-se a não movimentação do $\mathrm{P}$ ao longo do perfil do solo pelo fato de o Latossolo Bruno do presente estudo ser um solo intemperizado, com predominância de argila dos tipos caulinita, gibbsita, goethita e hematita (Martins, 2002), que, por apresentar elevado grau de adsorção específica do $\mathrm{P}$, dificulta a mobilização desse nutriente no perfil do solo (Novais \& Smyth, 1999; Souza et al., 2006).

Mudanças nos teores de $\mathrm{P}$, em profundidade, têm sido associadas ao uso de esterco por longos períodos ou em doses elevadas (Culley et al., 1981; Meek et al., 1982; Sharpley et al., 1984; Sutton et al., 1986; Chang et al., 1991; Eghball et al., 1996), indicando a importância do montante de $\mathrm{P}$ adicionado na movimentação. Meek et al. (1982), por exemplo, só 
Quadro 5. Efeito dos tratamentos de adubação orgânica e mineral sobre P-resina, P-Mehlich, K+, C-orgânico e condutividade elétrica, nas diversas profundidades do solo

\begin{tabular}{|c|c|c|c|c|c|c|c|}
\hline \multirow{3}{*}{ Variável-resposta } & \multirow{3}{*}{ Profundidade } & \multicolumn{5}{|c|}{ Efeitos } & \multirow{3}{*}{ CV } \\
\hline & & \multicolumn{2}{|c|}{ Mineral } & \multicolumn{2}{|c|}{ Esterco } & \multirow[t]{2}{*}{ Mineral x esterco } & \\
\hline & & $\mathbf{L}$ & $\mathbf{Q}$ & $\mathbf{L}$ & $\mathbf{Q}$ & & \\
\hline \multirow{6}{*}{ Fósforo resina $\left(\mathrm{mg} \mathrm{dm}^{-3}\right)$} & $\mathrm{cm}$ & & & & & & $\%$ \\
\hline & $0-5$ & $*$ & ns & $*$ & $\mathrm{~ns}$ & $\mathrm{~ns}$ & 18,29 \\
\hline & $5-10$ & * & $\mathrm{ns}$ & $\mathrm{ns}$ & $\mathrm{ns}$ & $\mathrm{ns}$ & 29,47 \\
\hline & $10-30$ & ns & $\mathrm{ns}$ & $\mathrm{ns}$ & $\mathrm{ns}$ & $\mathrm{ns}$ & 21,37 \\
\hline & $30-50$ & $\mathrm{~ns}$ & ns & $\mathrm{ns}$ & $\mathrm{ns}$ & $\mathrm{ns}$ & 37,91 \\
\hline & $50-80$ & $\mathrm{~ns}$ & $\mathrm{~ns}$ & $\mathrm{~ns}$ & $\mathrm{~ns}$ & $\mathrm{~ns}$ & 41,29 \\
\hline \multirow[t]{5}{*}{ Fósforo Mehlich-1 (mg dm-3) } & $0-5$ & * & $\mathrm{ns}$ & $*$ & $\mathrm{~ns}$ & $\mathrm{~ns}$ & 20,87 \\
\hline & $5-10$ & * & ns & $\mathrm{ns}$ & $\mathrm{ns}$ & $\mathrm{ns}$ & 33,57 \\
\hline & $10-30$ & ns & ns & $\mathrm{ns}$ & $\mathrm{ns}$ & $\mathrm{ns}$ & 26,20 \\
\hline & $30-50$ & $\mathrm{~ns}$ & ns & $\mathrm{ns}$ & $\mathrm{ns}$ & $\mathrm{ns}$ & 40,79 \\
\hline & $50-80$ & $\mathrm{~ns}$ & $\mathrm{~ns}$ & $\mathrm{~ns}$ & $\mathrm{~ns}$ & $\mathrm{~ns}$ & 46,60 \\
\hline \multirow[t]{5}{*}{ Potássio trocável $\left(\mathrm{cmol}_{\mathrm{c}} \mathrm{dm}^{-3}\right)$} & $0-5$ & ns & $\mathrm{ns}$ & * & $\mathrm{ns}$ & $\mathrm{ns}$ & 23,41 \\
\hline & $5-10$ & $\mathrm{~ns}$ & $\mathrm{~ns}$ & $*$ & $\mathrm{~ns}$ & $\mathrm{~ns}$ & 14,18 \\
\hline & $10-30$ & ns & $\mathrm{ns}$ & $*$ & $\mathrm{~ns}$ & $\mathrm{~ns}$ & 15,18 \\
\hline & $30-50$ & $\mathrm{~ns}$ & $\mathrm{~ns}$ & $*$ & $\mathrm{~ns}$ & $\mathrm{~ns}$ & 13,62 \\
\hline & $50-80$ & $\mathrm{~ns}$ & $\mathrm{~ns}$ & $*$ & $\mathrm{~ns}$ & $\mathrm{~ns}$ & 16,25 \\
\hline \multirow[t]{5}{*}{ Carbono orgânico (mg kg-1) } & $0-5$ & ns & $\mathrm{ns}$ & $*$ & $\mathrm{~ns}$ & $\mathrm{~ns}$ & 6,75 \\
\hline & $5-10$ & $\mathrm{~ns}$ & $\mathrm{~ns}$ & $\mathrm{~ns}$ & $\mathrm{~ns}$ & $\mathrm{~ns}$ & 7,78 \\
\hline & $10-30$ & $\mathrm{~ns}$ & $\mathrm{~ns}$ & $\mathrm{~ns}$ & $\mathrm{~ns}$ & $\mathrm{~ns}$ & 7,39 \\
\hline & $30-50$ & ns & $\mathrm{ns}$ & $\mathrm{ns}$ & $\mathrm{ns}$ & $\mathrm{ns}$ & 5,88 \\
\hline & $50-80$ & ns & ns & $\mathrm{ns}$ & $\mathrm{ns}$ & $\mathrm{ns}$ & 10,97 \\
\hline \multirow[t]{5}{*}{ Condutividade elétrica $\left(\mu \mathrm{S} \mathrm{cm}^{-1}\right)$} & $0-5$ & * & $\mathrm{ns}$ & $*$ & $\mathrm{~ns}$ & $\mathrm{~ns}$ & 26,78 \\
\hline & $5-10$ & $*$ & $*$ & * & $*$ & $\mathrm{~ns}$ & 33,79 \\
\hline & $10-30$ & $\mathrm{~ns}$ & $\mathrm{~ns}$ & $*$ & $\mathrm{~ns}$ & $\mathrm{~ns}$ & 18,55 \\
\hline & $30-50$ & $*$ & $\mathrm{~ns}$ & $*$ & $\mathrm{~ns}$ & $\mathrm{~ns}$ & 30,42 \\
\hline & $50-80$ & $*$ & $\mathrm{~ns}$ & $*$ & $\mathrm{~ns}$ & $\mathrm{~ns}$ & 48,32 \\
\hline
\end{tabular}

ns: não significativo a $5 \%$ pelo teste $\mathrm{F} ;{ }^{*}$ significativo a $5 \%$ pelo teste $\mathrm{F}$. **L: efeito linear; ***Q: efeito quadrático.

observaram aumento de P na camada de 30-60 cm quando foram utilizados aproximadamente $2.000 \mathrm{~kg} \mathrm{ha}^{-1}$ deste nutriente, enquanto Culley et al. (1981) obtiveram acréscimo nos teores de $\mathrm{P}$ na camada de 15 a $30 \mathrm{~cm}$ do solo, segunda camada avaliada, após aplicação de mais de $1.000 \mathrm{~kg} \mathrm{ha}^{-1} \mathrm{de} \mathrm{P}$ em um período de cinco anos. Esses valores ficaram muito acima da dose de $1.116 \mathrm{~kg} \mathrm{ha}^{-1}$ de $\mathrm{P}_{2} \mathrm{O}_{5}$ ou $480 \mathrm{~kg} \mathrm{ha}^{-1}$ de $\mathrm{P}$ aplicadas nos seis anos no presente trabalho. Logo, a característica do solo (alta capacidade de fixação), a forma de aplicação (aplicação em superfície) e as doses utilizadas, abaixo das relatadas na literatura supracitada, que constatou migração de $\mathrm{P}$, são as prováveis causas da baixa migração desse nutriente no solo.

Os teores de $\mathrm{K}$ no solo, após seis anos, ficaram muito abaixo do observado no início do experimento, e o teor deste mostrou-se indiferente às doses de adubação mineral aplicadas, em todas as profundidades avaliadas (Quadro 4). Na maioria dos trabalhos realizados na região Sul do Brasil, têm-se encontrado baixas respostas das culturas à adubação potássica (Sherer, 1998; Brunetto et al., 2005). Isso tem sido atribuído à contribuição de formas de K não trocáveis (Meurer et al., 1996; Melo et al., 2004), as quais se encontram em equilibrio com o K da solução do solo e suprem este elemento quando absorvido da solução (Martin \& Sparks, 1985; Richards et al., 1988; Nacthigall \& Vahl, 1991), pois, como os mecanismos que controlam a absorção de $\mathrm{K}$ nas plantas superiores são eficientes, principalmente em baixas concentrações na solução do solo, resulta em forte gradiente químico em direção à rizosfera (Gommers et al., 2005), criando um ambiente favorável à liberação do $\mathrm{K}$ de formas não trocáveis (Kaminski et al., 2007).

Diferentemente da adubação mineral, constatouse relação linear entre a dose de esterco e níveis de $\mathrm{K}$ disponível, em todas as profundidades analisadas (Figura 2). Essas diferenças entre os tipos de adubação no teor de $\mathrm{K}$ no solo certamente estão associadas ao montante de $\mathrm{K}$ adicionado via esterco, visto que esse nutriente, assim adicionado, supera a quantidade adicionada pela adubação mineral, no período de seis anos, em mais de três vezes (Quadros 2 e 3).

Em áreas de plantio direto regularmente adubadas é comum a estratificação de $\mathrm{K}$ ao longo do perfil. A não constatação de decréscimo gradual do $\mathrm{K}$ disponível 

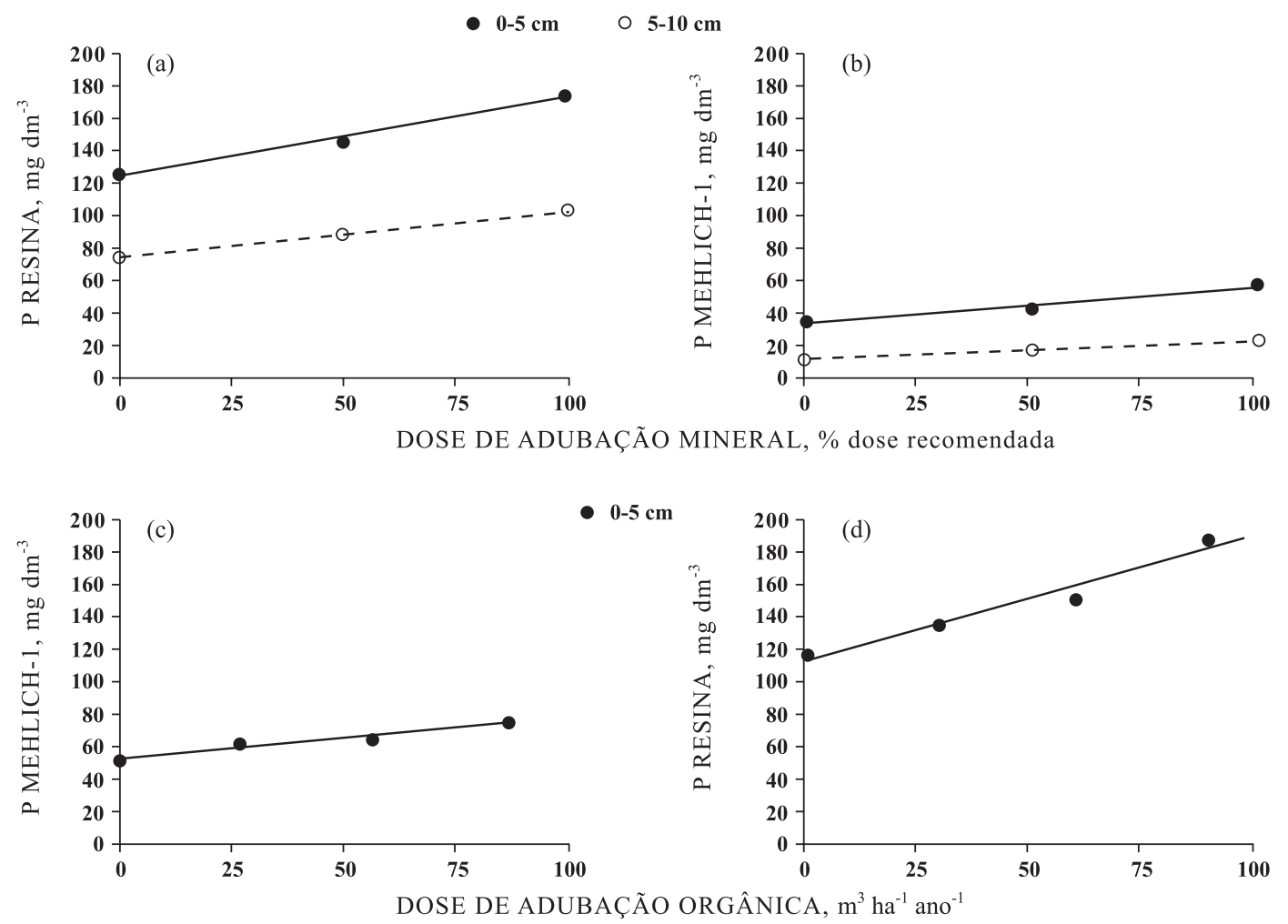

Figura 1. Teores de P-resina (a e c) e P Mehlich-1 (b e d) em sistema plantio direto submetido à adubação com diferentes doses de esterco líquido de gado leiteiro e adubação mineral, durante seis anos.

no perfil, combinada com baixos teores desse nutriente, quando da ausência ou uso de pequenas doses de esterco, sugere um esgotamento ao longo de todo o perfil do solo, provavelmente devido à produtividade bastante elevada das culturas e remoção da planta para produção de silagem, sem retorno do material vegetal ao solo, para que possa haver reciclagem dos nutrientes. Dessa forma, há esgotamento do K, em profundidade, pois existe exportação até 10 vezes mais alta de K pela silagem de milho que na produção de grãos (Vitosh et al., 1973).

Aumentos da disponibilidade e movimentação em profundidade estão condicionados ao balanço entre dose aplicada e exportação pelas culturas. Assim, o uso de elevadas doses pode aumentar $\mathrm{K}$ no solo em profundidade (Vitosh et al., 1973; Meek et al., 1982; Sutton et al., 1986) em área de produção de grãos, mas não garante o mesmo efeito sob produção de silagem, sobretudo quando o solo apresenta elevada capacidade de fixação de K (Vitosh et al., 1973; Culley et al., 1981).

Os resultados aqui observados corroboram os trabalhos de Warman (1987), que, usando esterco de gado de leiteiro em solos areno-argilosos no Canadá, também encontrou incremento nos níveis de $\mathrm{K}$ na profundidade de $0-15 \mathrm{~cm}$ usando $12 \mathrm{t} \mathrm{ha}^{-1}$ matéria seca, em experimento de dois anos. Diferentemente desse resultado, Elrashidi (1999) e Matsi et al. (2003) não verificaram aumento nos teores de $\mathrm{K}$ na superfície do solo após quatro anos de adição de esterco líquido de gado de leiteiro.

Os teores de $\mathrm{C}$ variaram de alto a médio, partindo da superfície até a profundidade de $80 \mathrm{~cm}$ (Comissão de Química e Fertilidade do Solo - CQFRS/SC, 2004), característica essa comum ao Latossolo Bruno. O teor de $\mathrm{C}$ foi alterado de modo significativo pelos tratamentos apenas na camada de $0-5 \mathrm{~cm}$, quando do uso de esterco, com uma relação linear entre as doses de esterco e teor de carbono (Figura 3). Essa

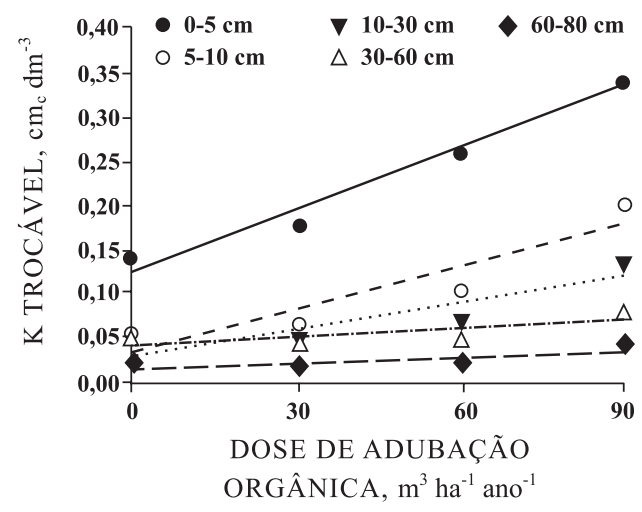

Figura 2. Teores de $\mathrm{K}^{+}$em sistema plantio direto submetido à adubação com diferentes doses de esterco líquido de gado leiteiro, durante seis anos. 
Quadro 6. Equações de regressão para teores de P-resina e P Mehlich-1, $\mathrm{K}^{+}$, carbono orgânico e condutividade elétrica em sistema plantio direto submetido a diferentes doses de esterco líquido de gado leiteiro e adubação mineral

\begin{tabular}{|c|c|c|c|c|c|}
\hline \multirow{2}{*}{ Variável-resposta } & \multirow{2}{*}{ Profundidade } & \multicolumn{2}{|l|}{ Fertilizante } & \multicolumn{2}{|l|}{ Esterco } \\
\hline & & Equação de regressão & $\mathbf{r}^{2}$ & Equação de regressão & $\mathbf{r}^{2}$ \\
\hline Fósforo resina $\left(\mathrm{mg} \mathrm{dm}^{-3}\right)$ & $\begin{array}{c}\mathrm{cm} \\
0-5 \\
5-10 \\
10-30 \\
30-50 \\
50-80\end{array}$ & $\begin{array}{l}\hat{y}=124,46+0,4775 X \\
\hat{y}=73,17+0,285 X \\
\hat{y}=45,92 \\
\hat{y}=14,75 \\
\hat{y}=6,00\end{array}$ & $\begin{array}{l}0,99 \\
0,99\end{array}$ & $\begin{array}{l}\hat{y}=113,93+0,7667 X \\
\hat{y}=87,42 \\
\hat{y}=45,92 \\
\hat{y}=14,75 \\
\hat{y}=6,00\end{array}$ & 0,95 \\
\hline Fósforo Mehlich-1 (mg dm-3) & $\begin{array}{c}0-5 \\
5-10 \\
10-30 \\
30-50 \\
50-80\end{array}$ & $\begin{array}{l}\hat{y}=35,758+0,2355 X \\
\hat{y}=14,304+0,0998 X \\
\hat{y}=8,04 \\
\hat{y}=3,02 \\
\hat{y}=2,11\end{array}$ & $\begin{array}{l}0,99 \\
0,99\end{array}$ & $\begin{array}{l}\hat{y}=36,643+0,242 X \\
\hat{y}=19,29 \\
\hat{y}=8,04 \\
\hat{y}=3,02 \\
\hat{y}=2,11\end{array}$ & 0,98 \\
\hline Potássio trocável $\left(\mathrm{cmol}_{\mathrm{c}} \mathrm{dm}^{-3}\right)$ & $\begin{array}{c}0-5 \\
5-10 \\
10-30 \\
30-50 \\
50-80\end{array}$ & $\begin{array}{l}\hat{y}=0,23 \\
\hat{y}=0,11 \\
\hat{y}=0,08 \\
\hat{y}=0,05 \\
\hat{y}=0,03\end{array}$ & & $\begin{array}{l}\hat{y}=0,1277+0,0023 X \\
\hat{y}=0,033+0,0016 X \\
\hat{y}=0,0343+0,0009 X \\
\hat{y}=0,0383+0,0003 X \\
\hat{y}=0,018+0,0002 X\end{array}$ & $\begin{array}{l}0,97 \\
0,86 \\
0,75 \\
0,68 \\
0,59\end{array}$ \\
\hline Carbono orgânico $\left(\mathrm{mg} \mathrm{kg}^{-1}\right)$ & $\begin{array}{c}0-5 \\
5-10 \\
10-30 \\
30-50 \\
50-80\end{array}$ & $\begin{array}{l}\hat{y}=40,56 \\
\hat{y}=30,36 \\
\hat{y}=24,87 \\
\hat{y}=27,90 \\
\hat{y}=20,43\end{array}$ & & $\begin{array}{l}\hat{y}=38,033+0,0561 x \\
\hat{y}=30,36 \\
\hat{y}=24,87 \\
\hat{y}=27,90 \\
\hat{y}=20,43\end{array}$ & 0,98 \\
\hline Condutividade eletrica ( $\mu \mathrm{s} \mathrm{cm}^{-1}$ ) & $\begin{array}{c}0-5 \\
5-10 \\
10-30 \\
30-50 \\
50-80\end{array}$ & $\begin{array}{l}\hat{y}=124,46+0,4775 X \\
\hat{y}=86,50-0,5275+0,0043 X^{2} \\
\hat{y}=61,25 \\
\hat{y}=53,625+0,0825 X \\
\hat{y}=46,708+0,1025 X\end{array}$ & $\begin{array}{l}0,99 \\
0,99 \\
0,99 \\
0,79\end{array}$ & $\begin{array}{l}\hat{y}=94,667+0,5389 X \\
\hat{y}=53,9+0,83 X-0,0043 X^{2} \\
\hat{y}=50,6+0,2367 X \\
\hat{y}=49,9+0,1744 X \\
\hat{y}=45,433+0,1422 X\end{array}$ & $\begin{array}{l}0,90 \\
0,92 \\
0,83 \\
0,89 \\
0,87\end{array}$ \\
\hline
\end{tabular}

relação pode estar associada uma ação direta da adição de $\mathrm{C}$ via esterco ou indireta pela elevação da adição de resíduo radicular, visto que ocorreu aumento da produtividade com uso de esterco, sendo este em geral maior que o adubo químico (Silva, 2005).

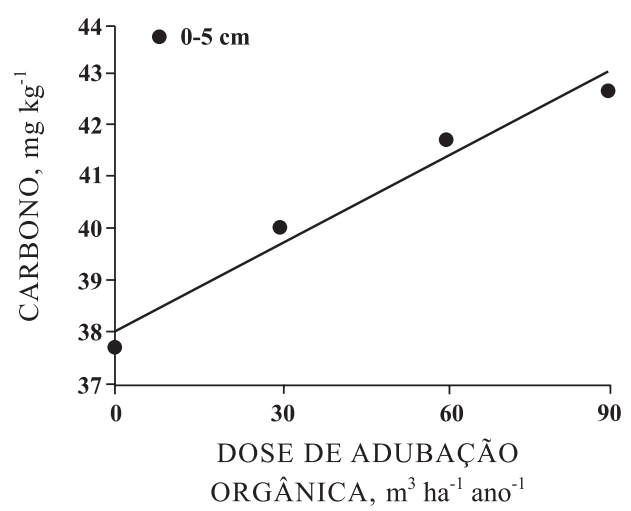

Figura 3. Teores de C-orgânico no solo submetido à adubação com diferentes doses de esterco líquido de gado leiteiro, durante seis anos.
Corroborando os resultados obtidos, acréscimos no teor de $\mathrm{C}$ pelo uso de esterco de gado têm sido frequentemente relatados (Tiarks et al., 1974; Meek et al., 1982; Ndayegamiye \& Cote, 1989; Sommerfeldt \& Chang, 1990; Chang et al., 1991), embora a ausência de efeito tenha sido constatada (Matsi et al., 2003). Contudo, maior acréscimo ou efeito significativo tem sido observado nas camadas mais superficiais do solo (Tiarks et al., 1974; Chang et al., 1991), sendo ele alterado principalmente pela dose aplicada (Culley et al., 1981; Sommerfeldt et al., 1986; Ndayegamiye \& Cote, 1989; Chang et al., 1991) e pelo tempo de aplicação (Sommerfeldt et al., 1986).

Vários fatores podem ter contribuído para a pequena elevação do teor de $\mathrm{C}$ de forma concentrada na camada superficial. O principal deles provavelmente tenha sido a retirada da fitomassa para silagem, o que resultou em deposição de pequena quantidade de resíduo sobre o solo. Elevado teor inicial de C no solo, dado pela condição natural e pelo fato de ele ter recebido resíduo por mais de 16 anos sem revolvimento, proveniente da criação intensiva de gado de corte antes da implantação do experimento, o que dificulta a retenção adicional do C aplicado (Ndayegamiye \& Coté, 1989). 
Os valores de condutividade elétrica obtidos em todas as profundidades são baixos quando comparados aos observados por Shortall \& Liebhardt (1975) e Chang et al. (1991), indicando a ocorrência de baixos teores de compostos ou de elementos minerais solúveis em solução. Esse fato é esperado, visto que baixo teor K disponível é um indicativo da elevada exportação deste nutriente pela silagem. Entretanto, a condutividade elétrica foi alterada em todas as profundidades avaliadas, apresentando relação linear com a aplicação de esterco líquido de gado leiteiro, com exceção da camada de $5-10 \mathrm{~cm}$, que obteve efeito quadrático (Quadro 5 e Figura 4).

Quanto à adubação mineral, houve efeito contrário ao da adubação com esterco até a profundidade de $30 \mathrm{~cm}$, havendo perda de condutividade elétrica na profundidade de $0-5 \mathrm{~cm}$, comportamento quadrático na profundidade de $5-10 \mathrm{~cm}$, queda na condutividade até uma dose de adubo mineral de $61,34 \%$ da dose recomendada, atingindo um nível mínimo de condutividade de $70,31 \mu \mathrm{S} \mathrm{cm}^{-1}$, com aumento da condutividade a partir deste nível de adubação. $\mathrm{Na}$ profundidade de $10-30 \mathrm{~cm}$, não houve efeito da adubação química, verificando-se comportamento semelhante ao da adubação com esterco nas profundidades abaixo de $30 \mathrm{~cm}$.

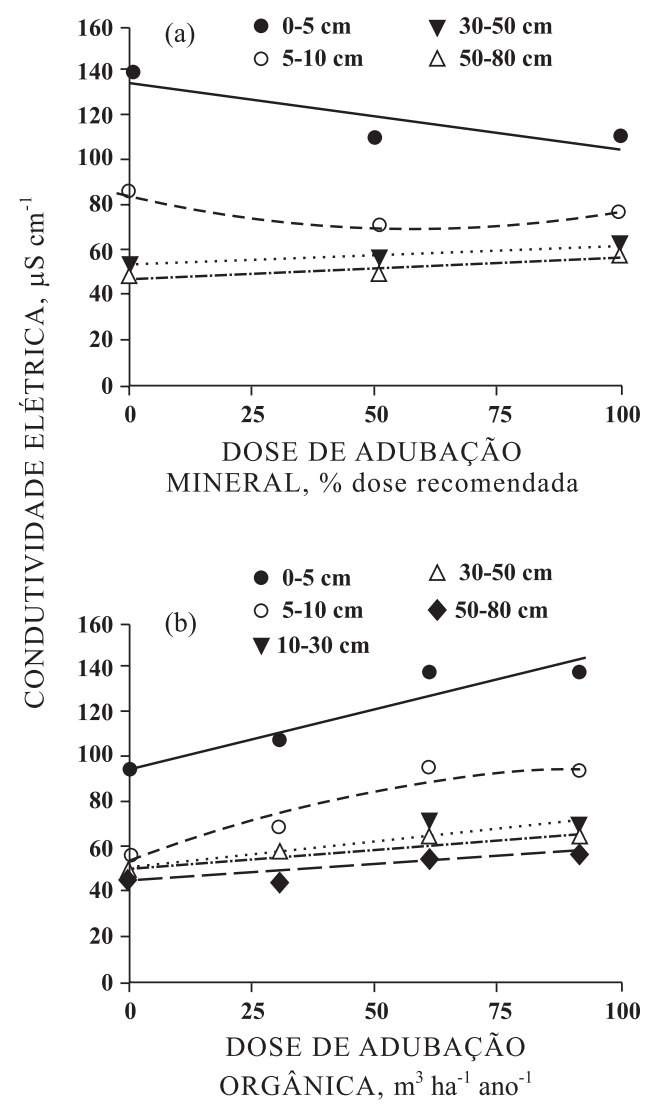

Figura 4. Condutividade elétrica em sistema plantio direto submetido à adubação com diferentes doses de esterco líquido de gado leiteiro (a) e adubação (b), durante seis anos.

\section{CONCLUSÕES}

1. As quantidades de esterco líquido de gado leiteiro e adubação mineral aplicadas na superfície do solo não alteram os níveis de $\mathrm{P}$ em profundidade, demonstrando a não ocorrência de migracão significativa de $\mathrm{P}$ no perfil do Latossolo Bruno.

2. A aplicação de esterco líquido de gado leiteiro aumenta os teores de K até a profundidade de $80 \mathrm{~cm}$; contudo, as quantidades aplicadas de esterco não foram suficientes para manter o $\mathrm{K}$ no perfil em níveis semelhantes ao inicial quando da implantação do experimento.

3. Embora os teores de C orgânico no solo sejam altos, a aplicação de esterco resultou em acúmulo adicional de $\mathrm{C}$, o qual foi restrito à camada superficial do solo.

4. A baixa condutividade elétrica ao longo do perfil sugere um esgotamento com retirada da planta inteira para silagem. A aplicação de esterco determinou maior valor de condutividade elétrica ao longo do perfil do solo.

\section{LITERATURA CITADA}

ASSMANN, T.S.; ASSMANN, J.M.; CASSOL, L.C.; DIEHL, R.C.; MANTELI, C. \& MAGIERO, E.C. Desempenho da mistura forrageira de aveia-preta mais azevém e atributos químicos do solo em função da aplicação de esterco. R. Bras. Ci. Solo, 31:1515-1523, 2007.

BRUNETTO, G.; GATIBONI, L.C.; RHEINHEIMER, D.S.; SAGGIN, A. \& KAMINSKI, J. Níveis críticos e resposta das culturas ao potássio em um Argissolo sob sistema plantio direto. R. Bras. Ci. Solo, 29:565-571, 2005.

CHANG, C.; SOMMERFELDT, T. G. \& ENTZ, T. Soil chemistry after eleven annual applications of cattle feedlot manure. J. Environ. Quality, 20:475-480, 1991.

COMISSÃO DE QUÍMICA E FERTILIDADE DO SOLO . CQFSRS/SC. Manual de adubação e de calagem para os estados do RS e de SC. Porto Alegre, Sociedade Brasileira de Ciência do Solo/Núcleo Região Sul, 2004. 400p.

CULLEY, J.L.B.; PHILLIPS, P.A.; HORE, F.R. \& PATNI, N.K. Soil chemical properties and removal of nutrients by corn resulting from different rates and timing of liquid dairy manure applications. Comm. J. Soil Sci., 61:35-46, 1981.

EGHBALL. B.; BINFORD, G.D. \& BALTENSPERGER, D.D. Phosphorus movement and adsorption in a soil receiving long-term manure and fertilizer application. J. Environ. Quality, 25:1339-1343, 1996.

ELRASHIDI, M.A. Mobility of elements in soil under stabilized dairy feedlot surfaces: A laboratory study. J. Environ. Quality, 28:1243-1251, 1999.

EMPRESA BRASILEIRA DE PESQUISA AGROPECUÁRIA EMBRAPA. Centro Nacional de Pesquisa de Solos. Sistema brasileiro de classificação de solos. Brasília, Embrapa. Produção de Informação; Rio de Janeiro, Embrapa Solos, 1999. 412p. 
FALLEIRO, R.M.; SOUZA, C.M.; SILVA, C.S.W.; SEDIYAMA, C.S.; SILVA, A.A. \& FAGUNDES, J.L. Influência dos sistemas de preparo nas profundidades químicas e físicas do solo. R. Bras. Ci. Solo, 27:1097-1104, 2003.

GEOHRING, L.D.; MCHUGH, O.V.; WALTER, M.T.; STEENHUIS, T.S.; AKHTAR, M.S. \& WALTER, M.F. Phosphorus transport into subsurface drains by macropores after manure applications: Implications for best management practices. Soil Sci., 166:896-909, 2001.

GOMMERS, A.; THIRY, Y. \& DELVAUX, B. Rhizospheric mobilization and plant uptake of radiocesium from weathered soils: I. Influence of potassium depletion. J. Environ. Quality, 34:2167-2173, 2005.

INSTITUTO AGRONÔMICO DO PARANÁ - IAPAR. Cartas climáticas do Estado do Paraná. Londrina, 2000. CD-ROM.

JOSAN, M.S.; NAIR, V.D.; HARRIS, W.G. \& HERRERA, D. Associated release of magnesium and phosphorus from active and abandoned dairy soils. J. Environ. Quality, 34:184-191, 2005.

KAMINSKI, J.; BRUNETTO, G.; MOTERLE, D.F. \& RHEINHEIMER, D.S. Depleção de formas de potássio do solo afetada por cultivos sucessivos. R. Bras. Ci. Solo, 31:1003-1010, 2007.

LEITE, L.F.C.; MENDONÇA, E.S.; NEVES, J.C.L.; MACHADO P.L.O.A. \& GALVÃO, J.C.C. Estoques totais de carbono orgânico e seus compartimentos em Argissolo sob floresta e sob milho cultivado com adubação mineral e orgânica. R. Bras. Ci. Solo, 27:821-832, 2003.

MARQUES, R. \& MOTTA, A.C.V. Análise química do solo para fins de fertilidade. In: Manual de diagnóstico da fertilidade e manejo dos solos agrícolas. 2.ed. Curitiba, Universidade Federal do Paraná, 2003. p.81-102.

MARTIN, H.W. \& SPARKS, D.L. On the behavior of nonexchangeable potassium in soils. Comm. Soil Sci. Plant Anal., 16:133-162, 1985.

MARTINS, R. Contribuição da reserva de potássio na nutrição e produção do trigo em solos do município de Castro, Estado do Paraná. Curitiba, Universidade Federal do Paraná, 2002. 94p. (Tese de Mestrado)

MATSI, T.; LITHOURGIDIS, A.S. \& GAGIANAS, A.A. Effects of injected liquid cattle manure on growth and yield of winter wheat and soil characteristics. Agron. J., 95:592596, 2003.

MEEK, B.; GRAHAM, L. \& DONOVAN, T. Long-term effects of manure on soil nitrogen, phosphorus, potassium, sodium, organic matter and water infiltration rate. Soil Sci. Soc. Am. J., 46:1014-1019, 1982.

MELO, G.W.; MEURER, E.J. \& PINTO, L.F.S. Fontes de potássio em solos distroférricos cauliníticos originados de basalto no Rio Grande do Sul. R. Bras. Ci. Solo, 28:597. 603, 2004.

MEURER, E.J.; KAMPF, N. \& ANGHINONI, I. Fontes potenciais de potássio em alguns solos do Rio Grande do Sul. R. Bras. Ci. Solo, 20:41-47, 1996.
MOTTA, A.C.V. Soil management impact soil quality in the Tennessee Valley of northern Alabama. Auburn, Auburn University, 2002. 153p.

NACHTIGALL, G.R. \& VAHL, L.C. Capacidade de suprimento de potássio dos solos da região Sul do Rio Grande do Sul. R. Bras. Ci. Solo, 15:37-42, 1991.

NDAYEGAMIYE, A. \& CÔTE, D. Effect of long-term pig slurry and solid cattle manure application on soil chemical and biological properties. Comm. J. Soil Sci., 69:39-47, 1989.

NOVAIS, R.F. \& SMYTH, T.J. Fósforo em solo e planta em condições tropicais. Viçosa, MG, Universidade Federal de Viçosa, 1999. 399p.

QUAGGIO, J.A. \& RAIJ, B.van. Comparação de métodos rápidos para determinação da matéria orgânica em solos. R. Bras. Ci. Solo, 3:184-187, 1979.

RAIJ, B.van.; QUAGGIO, J.A. \& SILVA, N.M. Extraction of phosphorus, potassium, calcium and magnesium from soils by in the ion-exchange resin procedure. Comm. Soil Sci. Plant Anal., 17:547-566, 1986.

RICHARDS, J.E. \& BATES, T.E. Studies on the potassiumsupplying capacities of Southern Ontario soils. II - nitric acid extraction nonexchangeable $\mathrm{K}$ and its availability to crops. Comm. J. Soil Sci., 63:199-208, 1988.

RYDEN, J.C.; SYERS, J.K. \& HARRIS, R.F. Phosphorus in runoff and streams. Adv.Agron., 25:1-45, 1973.

SCHJONNING, P.; CHRISTENSEN, B.T. \& CARSTENSEN, B. Physical and chemical properties of a sandy loam receiving animal manure, mineral fertilizer or no fertilizer for 90 years. Europ. J. Soil Sci., 45:257-268, 1994.

SEMEATO. Disponível em: <http://www.semeato.com.br>. Acesso em fev. de 2006

SHARPLEY, A.N.; SMITH, S.J.; STEWART, B.A. \& MATHERS, A.C. Forms of phosphorus in soil receiving cattle feedlot waste. J. Environ. Quality, 13:211-215, 1984.

SHERER, E.E. Níveis críticos de potássio para a soja em Latossolo húmico de Santa Catarina. R. Bras. Ci. Solo, 22:57-62, 1998.

SHERER, E.E.; BALDISSERA, I.T. \& NESI, C.N. Propriedades químicas de um Latossolo Vermelho sob plantio direto e adubação com esterco de suínos. R. Bras. Ci. Solo, 31:123$131,2007$.

SHORTALL, J.G. \& LIEBLARDT, W.C. Yield and growth of corn as affected by poultry manure. J. Environ. Quality, 4:186-191, 1975.

SILVA, J.C.P.M. Esterco líquido de gado de gado de leite e adubação mineral influenciando a produção de silagem e propriedades químicas do solo na região dos Campos Gerais do Paraná. Curitiba, Universidade Federal do Paraná, 2005. 63p. (Dissertação de Mestrado)

SIMS, J.T.; SIMARD, R.R. \& JOERN, B.C. Phosphorus loss in agricultural drainage: Historical perspective and current research. J. Environ. Quality, 27:277-293, 1998.

SOMMERFELDT, T.G. \& CHANG, C. Soil-waterproperties as affected by twelve annual applications of cattle feedlot manure. Soil Sci. Soc. Am. J., 49:7-9, 1986. 
SONNEVELD, C.; van den ENDE, J. \& BESS, S.S. Estimating the chemical composition of soil solutions by obtaining saturation extracts or specific $1: 2$ by volume extracts. Plant Soil, 122:169-175, 1990.

SOUZA, R.F.; FAQUIN, V.; TORRES, P.R.F. \& BALIZA, D.P. Calagem e adubação orgânica: Influência na adsorção de fósforo em solos. R. Bras. Ci. Solo, 30:975-983, 2006.

STATISTICAL ANALYSIS SYSTEM - SAS System for linear models. Cary: SAS Institute, 6 ed. 1996. 956p.

SUTTON, A.L.; NELSON, D.W.; KELLY, D.T. \& HILL, D.L. Comparison of solid dairy manure applications on corn yield and soil composition. J. Environ. Quality, 15:370$375,1986$.
TIARKS, A.E.; MAZURAK, A.P. \& CHESNIN, L. Physical and chemical properties of soil associated with heavy applications of manure from cattle feedlots. Soil Sci. Soc. Am. J., 38:826-830, 1974.

VITOSH, M.L.; DAVIS, J.F. \& KNEZEK, B.D. Long-term effects of manure, fertilizer, and plow depth on chemical properties of soils and nutrient movement in a monoculture corn system. J. Environ. Quality, 2:296-299, 1973.

WARMAN, P.R. The effects of pruning, fertilizers, and organic amendments on lowbush blueberry production. Plant Soil, 101:67-72, 1987. 
